\title{
Modelling Early Stages of Relativistic Heavy Ion Collisions: Coupling Relativistic Transport Theory to Decaying Color-electric Flux Tubes
}

\author{
M. Ruggieri, ${ }^{1}$ A. Puglisi, ${ }^{1,2}$ L. Oliva, ${ }^{1,2}$ S. Plumari, ${ }^{1,2}$ F. Scardina, ${ }^{1,2}$ and V. Greco ${ }^{1,2}$ \\ ${ }^{1}$ Department of Physics and Astronomy, University of Catania, Via S. Sofia 64, I-95125 Catania \\ ${ }^{2}$ INFN-Laboratori Nazionali del Sud, Via S. Sofia 62, I-95123 Catania, Italy
}

\begin{abstract}
In this study we model early times dynamics of relativistic heavy ion collisions by an initial color electric field which then decays to a plasma by the Schwinger mechanism, coupling the dynamical evolution of the initial color field to the dynamics of the many particles system produced by the decay. The latter is described by relativistic kinetic theory in which we fix the ratio $\eta / s$ rather than insisting on specific microscopic processes, and the backreaction on the color field is taken into account by solving self-consistently the kinetic and the field equations. We study isotropization and thermalization of the system produced by the field decay for a static box and for a $1+1 \mathrm{D}$ expanding geometry. We find that regardless of the viscosity of the produced plasma, the initial color electric field decays within $1 \mathrm{fm} / \mathrm{c}$; however in the case $\eta / s$ is large, oscillations of the field are effective along all the entire time evolution of the system, which affect the late times evolution of the ratio between longitudinal and transverse pressure. In case of small $\eta / s(\eta / s \lesssim 0.3)$ we find $\tau_{\text {isotropization }} \approx 0.8$ $\mathrm{fm} / \mathrm{c}$ and $\tau_{\text {thermalization }} \approx 1 \mathrm{fm} / \mathrm{c}$ in agreement with the common lore of hydrodynamics. Moreover we have investigated the effect of turning from the relaxation time approximation to the ChapmanEnskog one: we find that this improvement affects mainly the early times evolution of the physical quantities, the effect being milder in the late times evolution.
\end{abstract}

PACS numbers: 25.75.-q, 25.75.Ld, 25.75.Nq, 12.38.Mh

Keywords: Relativistic heavy ion collisions, Quark-gluon plasma, Relativistic Transport Theory, Schwinger Effect.

\section{INTRODUCTION}

The understanding of early times dynamics is one of the most interesting and compelling problems of heavy ion collisions at ultrarelativistic energy. According to the standard picture of such collision processes, immediately after the collision an ensemble of strong longitudinal color electric and color magnetic coherent fields known as Glasma is produced [1 [ [ ] $]$. Inhomogeneities in the Glasma fields are amplified by plasma instabilities, which eventually lead to loss of coherence of the color fields and to a locally isotropized particle plasma [7 21]. Recently the early isotropization problem has been studied also by means of AdS/CFT methods [22 26].

Besides plasma instabilities, a mechanism responsible for the initial field decays is the one introduced by Schwinger in the context of Quantum Electrodynamics [27, 28], known as the Schwinger effect which consists in a vacuum instability towards the creation of particle pairs by a strong electric field, and it is related to the existence of an imaginary part in the quantum effective action of a pure electric field, see [29] for a review. The problem of pair formation in strong electric fields has been considered recently by means of real time lattice simulations $30-32$. Moreover non abelian generalizations of the Schwinger production rate has been found for the case of both static and time dependent field [33 38]. In the context of quark-gluon plasma physics it has been considered as a mechanism for the color field decays in 39 59].

The purpose of the present study is to model early times dynamics of the system produced in relativistic heavy ion collisions by an initial color electric field which then decays to a plasma by the Schwinger effect. The focus of our study is to compute quantities which serve as indicators of thermalization and isotropization of the plasma. In particular, we couple the dynamical evolution of the color field to the dynamics of the many particles system produced by the decay, the latter being described by relativistic kinetic theory 6568 . As in the previous studies on this subject we assume that the dynamics of the color field is abelian, hence it satisfies the classical field Maxwell equations, see 56] for a review. Moreover in the present approach we neglect for simplicity initial longitudinal color magnetic fields which are present in the more complete description of the Glasma state. We consider two simple cases: the first one is a system evolving in a static box; then we consider a system with a longitudinal expansion which has a greater physical interest than the static box because it is closer to the picture of the early times dynamics of relativistic heavy ion collisions.

Besides being the first study in which a Monte Carlo method is used to simulate the Schwinger effect in the context of early times dynamics of high energy collisions, we improve previous studies which mainly rely on Relaxation Time Approximation (RTA) [54, 55] or on a linearization of the conductive electric current [45], by avoiding any ansatz both on the electric current and on the collision integral in the Boltzmann equation which permits to go beyond the RTA. The approach developed here, based on a stochastic solution of the relativistic Boltzman equation, has in perspective the advantage to be easily extended to $3+1 \mathrm{D}$ realistic simulations, allowing to study the possible impact of the early dynamics 
on observables like radial and elliptic flow 69 .

In both the cases of the static box and of the longitudinal expanding system we have focused on the calculation of $P_{L} / P_{T}$, where $P_{L}$ and $P_{T}$ denote the longitudinal and transverse pressures respectively. For the particular initial field configuration used in this work, namely an electric field along the longitudinal direction, one has $P_{L} / P_{T}=-1$; however the field decays onto particles thanks to the Schwinger effect and particle dynamics removes this initial anisotropy, the efficiency of isotropization being related to the coupling among the particles. In our simulations we fix the ratio $\eta / s$ where $\eta$ is the shear viscosity and $s$ the entropy density, and compute locally the corresponding cross section by means of the Chapman-Enskog (CE) approximation [65, 66]. We find that increasing $\eta / s$ the system is less efficient in removing the initial anisotropy in agreement with previous studies, although regardless of $\eta / s$ the longitudinal pressure becomes positive within a small fraction of $\mathrm{fm} / \mathrm{c}, \tau \approx 0.1$ $\mathrm{fm} / \mathrm{c}$; moreover for large values of $\eta / s$ we find persistent oscillations of the color field which affects the evolution of physical quantities like $P_{L} / P_{T}$ and the proper energy density, in agreement with [54, 55]. Even if we go beyond the RTA we find that only the early times evolution is affected, leaving the main behaviours very mildly affected.

The plan of the article is as follows. In Section II we review briefly the abelian flux tube model which we implement in our simulations. In Section III we review relativistic transport theory. In Section IV we present our results of the simulations for the case of a static box. In Section $\mathrm{V}$ we discuss in detail our results for the case of a box with a longitudinal expansion. Finally in Section VI we draw our conclusions.

\section{ABELIAN FLUX TUBE MODEL}

In this Section we briefly summarize the abelian flux tube model (AFTm) 39 56] which we implement in our simulations of the initial stage of relativistic heavy ion collisions. In the present study we do not insist on implementing the most realistic geometrical condition relevant for heavy ion collisions experiments, in which one should take into account several flux tubes in the transverse plane similar to the glasma configuration; rather we consider a simpler situation in which there is only one flux tube of a given transverse area, and study its dynamical evolution by coupling the field equations to relativistic kinetic theory for the particle quanta produced by the decay of the field itself, leaving to upcoming works the study of more realistic initial conditions.

The main assumptions of the AFTm are:

- In the initial condition a color electric field is present, which is produced by color charges in the colliding nuclei;

- The color electric field decays into particle quanta by the Schwinger mechanism;
- The particle quanta propagate in the medium colliding and interacting with the background of the color field;

- Particle creation as well as particle currents affect in a self-consistent way the color electric field;

- The field dynamics is abelian, namely it satisfies the abelian Maxwell equations.

The last assumption is quite strong because initial gauge fields are quite large, nevertheless it permits the easiest implementation of the coupling of classical field equations to relativistic kinetic theory in presence of the Schwinger mechanism, hence we will limit ourselves to consider abelian dynamics of the classical fields leaving the introduction of non abelian field dynamics to future works.

We will discuss more about kinetic theory in the next Section, therefore in this Section we mainly focus on the Schwinger mechanism which is responsible for particle production in the early stage of the collision, and on the Monte Carlo implementation we use to simulate the Schwinger effect.

In this Section we closely follow 54 at the same time adopting a slightly different notation which is more convenient for this article. Moreover, since the very beginning we assume the initial color field to be polarized along the $3^{r d}$ direction of adjoint color space, meaning that only one particular color charge is present in the two colliding nuclei [54]. Moreover we assume only one component of the electric field is present, namely the one in the longitudinal direction, which we denote by $E$ in the following. The latter is a consequence of the fact that in the initial condition we assume the field is purely longitudinal, and transverse currents are not produced during time evolution of the system if transverse expansion does not take place implying a vanishing transverse field. The number of pairs per unit of spacetime and invariant momentum space produced by the decay of the electric field by the Schwinger effect is (we assume massles quanta throughout this article)

$$
\frac{d N_{j c}}{d \Gamma} \equiv p_{0} \frac{d N_{j c}}{d^{4} x d^{2} p_{T} d p_{z}}=\mathcal{R}_{j c}\left(p_{T}\right) \delta\left(p_{z}\right) p_{0},
$$

with

$$
\mathcal{R}_{j c}\left(p_{T}\right)=\frac{\mathcal{E}_{j c}}{4 \pi^{3}}\left|\ln \left(1 \pm e^{-\pi p_{T}^{2} / \mathcal{E}_{j c}}\right)\right|
$$

the plus (minus) sign corresponding to the creation of a boson (fermion-antifermion) pair. In this equation $p_{T}$, $p_{z}$ refer to each of the two particles created by the tunneling process; $\mathcal{E}_{j c}$ is the effective force which acts on the tunneling pair and it depends on color and flavor; it can be written as

$$
\mathcal{E}_{j c}=\left(g\left|Q_{j c} E\right|-\sigma_{j}\right) \theta\left(g\left|Q_{j c} E\right|-\sigma_{j}\right),
$$

where $\sigma_{j}$ denotes the string tension depending on the kind of flavor considered. Moreover $p_{0}=\sqrt{p_{T}^{2}+p_{z}^{2}}$ corresponds to the single particle kinetic energy. 
The $Q_{j c}$ are color-flavor charges which, in the case of quarks, correspond to the eigenvalues of the $T_{3}$ operator:

$$
Q_{j 1}=\frac{1}{2}, \quad Q_{j 2}=-\frac{1}{2}, \quad Q_{j 3}=0, \quad j=1, N_{f} ;
$$

for antiquarks, corresponding to negative values of $j$, the color-flavor charges are just minus the corresponding charges for quarks. Finally for gluons (which in our notation correspond to $j=0$ ) the charges are obtained by building gluons up as the octet of the $3 \otimes \overline{3}$ in color space; in particular

$$
Q_{01}=1, \quad Q_{02}=\frac{1}{2}, \quad Q_{03}=-\frac{1}{2},
$$

and $Q_{04}=-Q_{01}, Q_{05}=-Q_{02}, Q_{06}=-Q_{03}$. The above charges are obtained easily [56]: for example, the gluon we label with subscript 1 can be represented, from the point of view of transformations in color space, as a redantigreen state; its $T_{3}$ charge is then given by difference of $T_{3}$ charges of a red and a green state. We notice that we have only six gluons out of eight, corresponding to the non-diagonal color generators; the gluon fields corresponding to the diagonal color generators have vanishing coupling with the background field, hence they cannot be produced by the Schwinger effect.

As a final remark we would like to observe that, even if the model is named abelian, such nomenclature simply refers to the fact that in the evolution equation for the classical field, self-interacion terms coming from non vanishing structure constants of the color gauge group are neglected [56]. However interactions among the classical field and gluons are still present in this calculations, thanks both to the Schwinger effect which produces charged gluons, and to conduction currents which affect the evolution of the field, see the next Section for more details.

\section{RELATIVISTIC TRANSPORT THEORY COUPLED TO MAXWELL EQUATIONS}

Our calculation scheme is based on the Relativistic Transport Boltzmann equation which, in the presence of a gauge field $F^{\mu \nu}$, can be written as follows:

$$
\left(p^{\mu} \partial_{\mu}+g Q_{j c} F^{\mu \nu} p_{\nu} \partial_{\mu}^{p}\right) f_{j c}(x, p)=\frac{d N_{j c}}{d \Gamma}+\mathcal{C}_{j c}[f]
$$

where $f_{j c}(x, p)$ is the distribution function for flavour $j$ and color $c, F^{\mu \nu}$ is the electromagnetic tensor. On the right hand side we have the source term $d N / d \Gamma$ which describes the creation of quarks, antiquarks and gluons due to the decay of the color electric field and $\mathcal{C}[f]$ which represents the collision integral. Considering only $2 \rightarrow$ 2 body elastic scatterings, the collision integral can be written as:

$$
\begin{aligned}
\mathcal{C}[f]= & \int \frac{d^{3} p_{2}}{2 E_{2}(2 \pi)^{3}} \frac{d^{3} p_{1^{\prime}}}{2 E_{1^{\prime}}(2 \pi)^{3}} \frac{d^{3} p_{2}^{\prime}}{2 E_{2}^{\prime}(2 \pi)^{3}}\left(f_{1^{\prime}} f_{2^{\prime}}-f_{1} f_{2}\right) \\
& \times|\mathcal{M}|^{2} \delta^{4}\left(p_{1}+p_{2}-p_{1^{\prime}}-p_{2^{\prime}}\right),
\end{aligned}
$$

where we omit flavour and color indices for simplicity, $\mathcal{M}$ is the transition matrix for the elastic process linked to the differential cross section through $|\mathcal{M}|^{2}=16 \pi s^{2} d \sigma / d t$, being $s$ the Mandelstam variable.

In our simulations we solve numerically Eq. (6) using the test particles method and the collision integral is computed using Monte Carlo methods based on the stochastic interpretation of transition amplitude 60 65, 67, 68].

The evolution of the electric field is given by the Maxwell equations:

$$
\frac{\partial E}{\partial z}=\rho, \quad \frac{\partial E}{\partial t}=-j
$$

where $\rho$ corresponds to the color charge density,

$$
\rho=g \sum_{j, c} Q_{j c} \int d^{3} \boldsymbol{p} f_{j c}(p),
$$

where $j, c$ denote flavor and color respectively; the sum in the above equation runs over quarks, antiquarks and gluons. On the other hand $j$ corresponds to the color electric current which is given by the sum of two contributions: in fact the Schwinger effect can be described as a dielectric breakdown in which dipoles are produced by quantum tunneling hence changing the local dipole moment of the vacuum, and the charges move in the medium due to the residual electric field giving rise to a conductive current. Following [54] we name displacement current, $j_{D}$, and matter current, $j_{M}$ :

$$
j=j_{M}+j_{D} .
$$

Here $j_{M}$ is a colored generalization of the usual electric current density which in a continuum notation is given by

$$
j_{M}^{\mu}=g \sum_{j, c} Q_{j c} \int \frac{d^{3} \boldsymbol{p}}{p_{0}} p^{\mu} f_{j c}(p) .
$$

The displacement current arises from the polarization of the vacuum due to the decay of the electric field by the Schwinger mechanism: more precisely it is given by the time derivative of the local dipole moment induced by the particles pop-up, in the same way a time variation of the local dipole moment in a medium gives rise to a change in the local electric field [71]. According to the quantum tunneling interpretation of the Schwinger effect [39] the dipole moment can be computed as $2 p_{0} / E$ where $p_{0}$ corresponds to the kinetic energy of the particles coming out from the vacuum; taking into account Eq. (1) $j_{D}$ can be written, in the reference frame where particles are produced with vanishing longitudinal momentum, as

$$
j_{D}=\sum_{j=0}^{N_{f}} \sum_{c=1}^{3} \int \frac{d^{3} \boldsymbol{p}}{p_{0}} \frac{d N_{j c}}{d \Gamma} \frac{2 p_{T}}{E}
$$

where $N_{f}$ corresponds to the number of flavors in the calculation. The color charge and current densities depend 
on the particle distribution function: hence they link the Maxwell equations (8) to the kinetic equation (6). We solve self-consistently the field and kinetic equations: in this way we take into account the back reaction of particle production and propagation on the color field.

At variance with the standard use of transport theory, in which one fixes a set of microscopic processes into the collision integral, we have developed an approach that fixes the total cross section in order to have the wanted $\eta / s$. By means of this scheme we are able to use the Boltzmann equation to simulate the dynamical evolution of a fluid with specified shear viscosity, in analogy to what is done within hydrodynamical simulations.

We use the CE approach to relate shear viscosity to temperature, cross section and density which is in agreement with Green-Kubo correlator results 66. Therefore, we fix $\eta / s$ and compute the pertinent total cross section by mean of the relation

$$
\sigma_{t o t}=\frac{1}{5} \frac{T}{\rho g(a)} \frac{1}{\eta / s},
$$

which is valid for a generic differential cross section $d \sigma / d t \sim \alpha_{s}^{2} /\left(t-m_{D}^{2}\right)^{2}$ as proved in [66]. In the above equation $a=m_{D} / 2 T$, with $m_{D}$ the screening mass regulating the angular dependence of the cross section, while

$$
g(a)=\frac{1}{50} \int d y y^{6}\left[\left(y^{2}+\frac{1}{3}\right) K_{3}(2 y)-y K_{2}(2 y)\right] h\left(\frac{a^{2}}{y^{2}}\right)
$$

where $K_{n}$ is the Bessel function and the function $h$ relates the transport cross section to the total one $\sigma_{t r}(s)=$ $\sigma_{\text {tot }} h\left(m_{D}^{2} / s\right)$ being $h(\zeta)=4 \zeta(1+\zeta)[(2 \zeta+1) \ln (1+1 / \zeta)-$ $2]$. The $g(a)$ is the proper function accounting for the correct relaxation time $\tau_{\eta}^{-1}=g(a) \sigma_{\text {tot }} \rho$ associated to the shear viscosity transport coefficient. For isotropic cross section, i. e. $m_{D} \rightarrow \infty$, the function $g(a)$ is equal to $2 / 3$ and Eq. 13 reduces to the relaxation time approximation with $\tau_{\eta}^{-1}=\tau_{t r}^{-1}=\sigma_{t r} \rho$, while for finite value of $m_{D}$, which means anisotropic scatterings, $g(a)<2 / 3$.

We notice that, in the regime where viscous hydrodynamics applies, the specific microscopic details of the cross section are irrelevant, and our approach is an effective way to employ transport theory to simulate a fluid at a given $\eta / s[68,72]$.

\section{FLUX TUBE DECAY IN A STATIC BOX}

In this Section we study the chromoelectric flux tube decay in a static box. We assume the box is a cube with side of $5 \mathrm{fm}$. Moreover we assume periodic boundary conditions for particles propagating in the box. This case is of academic interest, nevertheless it is useful because it allows us to introduce concepts which will be useful when we will consider more interesting case of a longitudinally expanding background, as well as it provides a further test of the numerical solution of Eq. (6).
Given the symmetry of the problem, assuming at initial time a homogeneous electric field along the $z$ direction, then the system will remain homogeneous along the whole dynamical evolution: the electric field at later times as well as the currents and the invariant distribution functions will depend only on time and not on space coordinates. Moreover it is easy to verify by Maxwell equations that neither magnetic fields nor transverse components of the electric field can develop during the evolution. Within these assumptions the evolution equation for the classical field relevant for our problem is given by

$$
\frac{d E}{d t}=-j(t)
$$

In our simulations in the static box case we use Eq. (1) to create particle pairs from the color electric field: at each time step, the value of $E$ and of the volume box being given, we compute the expected pair number, $\mathcal{N}$, integrating Eq. (11) over the volume box, then we distribute the $\mathcal{N}$ pairs uniformly in the box and with transverse momentum $p_{T}$ according to the distribution in Eq. (11); since the pairs have to pop out from the vacuum with vanishing total and longitudinal momenta, given $p_{T}$ we extract randomly the azimuthal angle $\phi$ which uniquely determines $p_{y}=p_{T} \sin \phi$ and $p_{x}=p_{T} \cos \phi$ of one of the particles in the pair; finally the momentum direction of the second particle is given by $\pi-\phi$.

In Fig. 1 we plot the early times evolution of the colorelectric field for the cases of calculations at fixed total cross section (upper panel) as well as fixed $\eta / s$ (lower panel). In the upper panel of the figure the line labelled as ideal gas means a calculation with zero cross section.

From the results in the upper panel of Fig. 1 we notice that for small coupling among the particles, the electric field rapidly decays through the Schwinger mechanism then it evolves with damped oscillations: the smaller the coupling is, the less efficient the damping is. Eventually for strong enough coupling the oscillations disappear and the color-electric fields just decays according to a power law. This dependence on the coupling strength is very easy to understand and is in last analysis due to the dependence of the electric conductivity of the plasma on the particle interaction strength 70 . In fact at initial times the particles are produced by the Schwinger effect with zero longitudinal momentum, then in case the coupling is small they are accelerated by the electric field thus generating an electric current $j_{M}$ which at first gains energy from the field thus lowering its magnitude; because of the small coupling particles's momenta is not randomized thus currents are efficiently produced by the field. At some point of time the field is zero but the current $j_{M}$ is still nonvanishing and positive hence causing a sign flip of the electric field and a negative acceleration of the charges, resulting eventually in $j_{M}<0$ and an increase of the electric field. This process causes the field oscillations we observe in Fig. 1]

On the other hand, for large values of the coupling the 

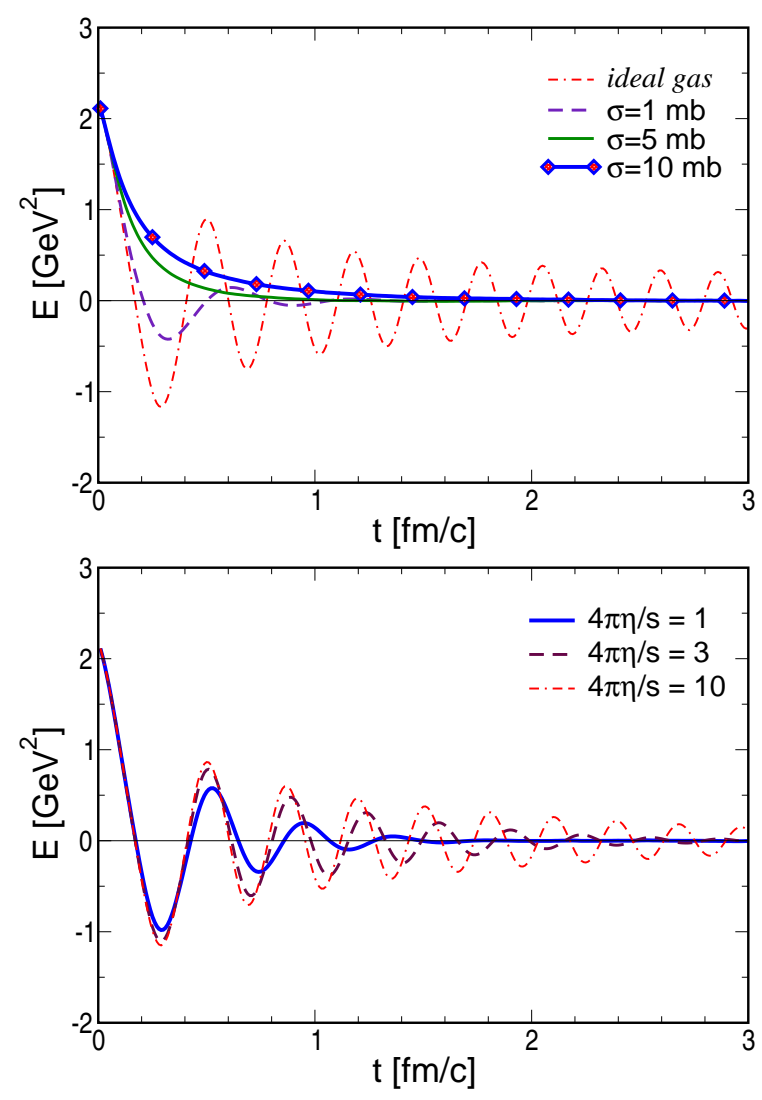

FIG. 1: Early times evolution of the color-electric field for the cases of calculations at fixed total cross section (upper panel) as well as fixed $\eta / s$ (lower panel).

scattering processes among the particles randomize momenta hence causing $j_{M} \approx 0$ and the time evolution of the field is a pure decay by the Schwinger effect. To consider this point more closely we write down the analytical form of Equation (12), namely

$$
j_{D}=\frac{\zeta(5 / 2)}{4 \pi^{3} E}\left[\frac{4-\sqrt{2}}{4} \sum_{c=1}^{3} \mathcal{E}_{0 c}^{5 / 2}+\sum_{j=1}^{N_{f}} \sum_{c=1}^{3} \mathcal{E}_{j c}^{5 / 2}\right]
$$

For example in the case one considers only the decay into gluons and assuming for simplicity $\sigma_{g}=0$ in Eq. (3) one has for $j_{M}=0$ the equation

$$
\frac{d E}{d t}=-\sigma_{D} E^{3 / 2}
$$

where we have introduced the quantity

$$
\sigma_{D}=\frac{\zeta(5 / 2)}{4 \pi^{3}} \frac{4-\sqrt{2}}{4} \sum_{c=1}^{3}\left|g Q_{0 c}\right|^{5 / 2},
$$

which is related to the Schwinger effect and is independent on the coupling among the particles. The solution of Eq. (17) can be found as

$$
E=\frac{4 E(0)}{\left(2+\sigma_{D} \sqrt{E_{0}} t\right)^{2}},
$$

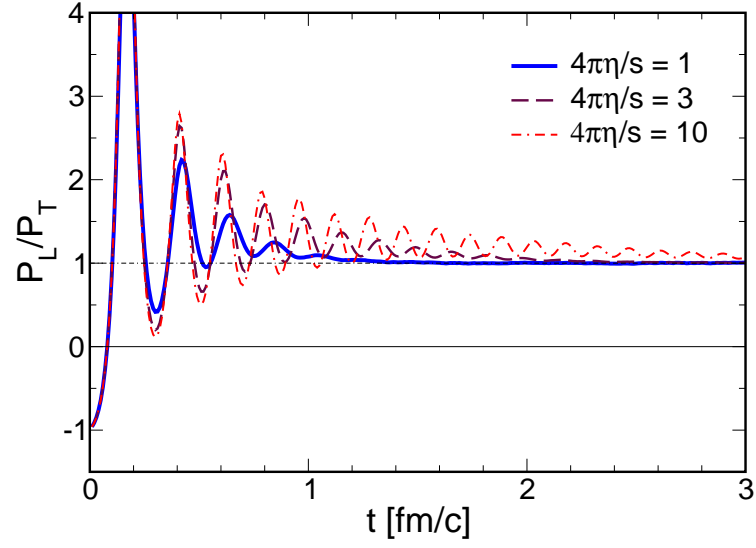

FIG. 2: Time evolution of the ratio $P_{L} / P_{T}$ for the static box, for several values of $\eta / s$.

showing that in absence of a conductive current the decay of the chromoelectric field is purely power law.

In the lower panel of Fig. 1 we plot the early times evolution of the color electric field keeping $\eta / s$ fixed. The calculations have been performed assuming an isotropic cross section, and the relation among the total cross section and $\eta / s$ is computed by the CE method 66 which, as proved in 66], is in agreement with the relaxation time approximation for the case of an isotropic differential cross section. From the qualitative point of view these results do not differ from those we obtain for calculations at fixed cross section, and they can be understood in the same way the results we obtained in the case of a fixed cross section.

In Fig. 2 we plot the time evolution of the ratio $P_{L} / P_{T}$ for the case of the static box, for several values of $\eta / s$. As expected we find that for small values of $\eta / s$ the system is very efficient in removing the initial anisotropy. We notice that the initial longitudinal pressure is negative and $P_{L} / \varepsilon=-1$; indeed at initial time the system is made of pure color electric field for which $T^{\mu \nu}=\operatorname{diag}\left(\varepsilon, P_{T}, P_{T}, P_{L}\right)$ with $\varepsilon=E^{2} / 2, P_{T}=\varepsilon$ and $P_{L}=-\varepsilon$. On the other hand as soon as particles are produced by the Schwinger effect, they give a positive contribution to the longitudinal pressure and the field magnitude decreases, eventually leading to a positive pressure. Moreover because of the strong interactions among the particles produced, $P_{L}$ and $P_{T}$ tend to the same value within a very short time range, $\tau_{\text {iso }} \approx 1$ $\mathrm{fm} / \mathrm{c}$.

We notice that regardless of the value of $\eta / s$ the system tends to remove the initial pressure anisotropy, which is quite natural in the case of the static box since collisions always lead to the equilibrium state. However isotropization times are different and indeed in the case of a weakly coupled system, see for example the case $4 \pi \eta / s=10$ in Fig. 2. the equilibrium state is reached in a much larger time than in the case of $4 \pi \eta / s=1$. Moreover the oscillations of the electric field strength in Fig. 1 lead to 
many oscillations of $P_{L} / P_{T}$ in the case $4 \pi \eta / s=10$, such oscilations being damped effectively within the first $\mathrm{fm} / \mathrm{c}$ in the case $4 \pi \eta / s=1$.

\section{FLUX TUBE WITH BOOST-INVARIANT LONGITUDINAL EXPANSION}

In this Section we study the effect of a boost invariant longitudinal expansion on pair production from the decay of a chromoelectric flux tube. We assume the expansion takes place along the direction of the electric field; moreover the dynamics is invariant for boosts along the longitudinal direction. For this case we will discuss more results because it is closer to the description of the central rapidity region in the early stages of a relativistic heavy ion collision. Once again we closely follow the formalism of [54].

We remind very quickly the relevant equations in this case. The evolution equations for the color electric field are given by the pair of Maxwell equations

$$
\frac{\partial E}{\partial z}=\rho, \quad \frac{\partial E}{\partial t}=-j,
$$

where both current and charge densities are computed in the laboratory frame. Assuming boost invariance along the longitudinal direction implies that $\mathcal{E}_{z}$ depends only on proper time $\tau=\sqrt{t^{2}-z^{2}}$. We can combine the two Eqs. (20) to form a boost invariant equation, namely

$$
\tau \frac{d E}{d \tau}=z \rho-t j
$$

which can be rewritten as

$$
\frac{d E}{d \tau}=\rho \sinh \eta-j \cosh \eta
$$

where the right hand side corresponds to the (minus) electric current computed in the reference frame where the time is the proper time, namely the local rest frame of the fluid. Equation (22) is in agreement with the boost invariant form of Maxwell equation used in [54], see also [45].

To solve Eq. (22) we adopt a finite difference scheme and prepare a box with a square cross section in the transverse direction, with $-x_{\max } \leq x \leq x_{\max }$ and $-y_{\max } \leq y \leq y_{\max }$, and with cells in space-time rapidity, fixing the range of $\eta$ in which we distribute the produced particles by $-\eta_{\max } \leq \eta \leq \eta_{\max }$ with $\eta_{\max }=2.5$. This implementation corresponds to have a box with a longitudinal expansion since from the well known equations of relativistic kinematics one gets $z_{\max }=t \tanh \eta_{\max }$ which, for $\eta_{\max }$ sufficiently large, corresponds to a wall moving at ultrarelativistic speed along the longitudinal direction. We therefore distribute the pairs created by the decay of the flux tube with uniform probability in each of the cells in $(x, y, \eta)$.

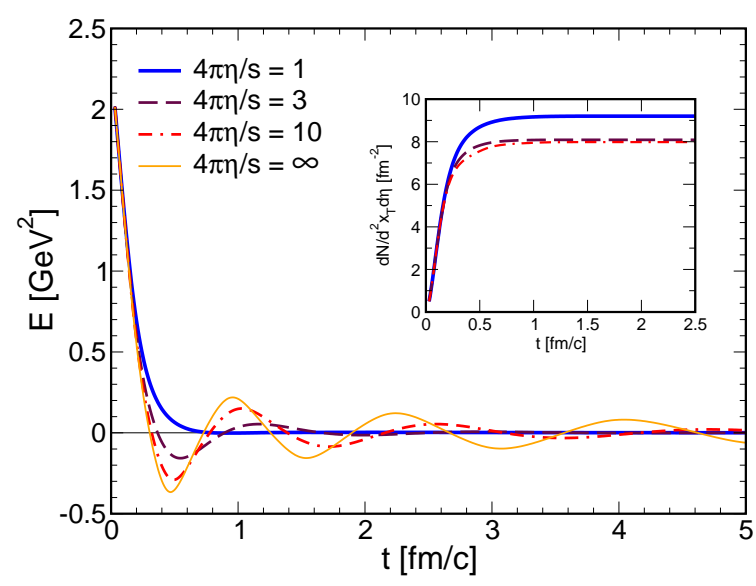

FIG. 3: Chromoelectric field strength (main panel) and particle number produced per unit of transverse area and rapidity (inset panel) as a function of time. The electric field is averaged in the central rapidity region $|\eta|<0.5$.

To take into account the longitudinal expansion Eq. (10) has to be modifed as 54

$$
\frac{d N}{d^{4} x d^{2} p_{T} d y}=\mathcal{R}\left(p_{T}\right) \delta(w) v,
$$

where $y$ denotes the momentum rapidity; $\mathcal{R}\left(p_{T}\right)$ depends only on transverse momentum and it is not affected formally by the expanding geometry; moreover we have introduced the two boost-invariant variables

$$
w=t p_{z}-z p_{0}, \quad v=p_{0} t-z p_{z},
$$

and $\delta(w)$ affects the longitudinal momentum distribution by forcing the condition

$$
p_{z}=\frac{z}{t} p_{0}=p_{T} \sinh \eta
$$

for the produced pairs, with $p_{0}=\sqrt{p_{T}^{2}+p_{z}^{2}}$; such a condition is equivalent to assume that momentum rapidity of the produced pair is equal to space-time rapidity. The procedure we implement to create pairs in the case of the boost invariant longitudinal expansion is very similar to the one we have described in the case of the static box, the only difference being that in the present case we iterate the static box procedure for each rapidity cell.

\section{A. Field decay, particle production and spectra}

In Fig. 3 we plot the color electric field strength averaged in the central rapidity region $|\eta|<0.5$ (main panel) and particle number produced per unit of transverse area and rapidity (inset panel) as a function of time. The calculations are shown for three different values of $\eta / s$ (calculations at fixed total cross section give similar results); the relation among the total cross section and $\eta / s$ 
we have used in the simulation is the $\mathrm{CE}$ relation with an isotropic cross section, see Eqs. (13) and (14). The electric field is averaged in the central rapidity region $|y|<0.5$ because in this region within a $10 \%$ of accuracy one has $t \approx \tau$ with $t$ corresponding to the laboratory frame in which the system expands, so a comparison with 54] where the dynamics is followed in $\tau$ is more meaningful. The initial value we have used in the simulations is $E(t=0)=2.2 \mathrm{GeV}^{2}$ in agreement with the LHC case of 54] but we obtain similar results by changing this value.

As for the case of the static box we find that for the case of boost invariant longitudinal expansion the chromoelectric field experiences a rapid decay for small values of $\eta / s$. Once again this is due to the fact that in this case the coupling among particles is large, meaning collisions are very effective in randomizing particle momenta in each cell hence damping conductive currents that might sustain the field. On the other hand for intermediate and large values of $\eta / s$ the electric field experiences stronger fluctuations during time evolution.

In the inset of Fig. 3 we plot the number of produced gluons per unit of transverse area and rapidity versus time. We find that regardless of the value of $\eta / s$ we use in the simulation, the particles are produced at very early times, approximately within $0.5 \mathrm{fm} / \mathrm{c}$, with the only expection of very few particles produced at later times in the case $4 \pi \eta / s=10$. We have checked that changing the initial value $E_{0}$ of the electric field does not modify the production time in a considerable way unless $E_{0}$ is very small, namely $E_{0} \ll 1 \mathrm{GeV}^{2}$. Moreover the value of $\eta / s$ affects the conversion of the initial classical field to gluons only within a few percent: for example comparing the results for $4 \pi \eta / s=1$ and $4 \pi \eta / s=3$ we find in the latter case a lowering of less than $10 \%$ on the number of particles produced.

In the upper panel of Fig. 4 we plot the proper kinetic energy density, $\varepsilon_{k i n}$, at central rapidity $(|\eta|<0.5)$, versus laboratory time for three different values of $\eta / s$ (calculations at fixed total cross section give similar results). The relation among $\eta / s$ and cross section is fixed by the CE relation with an isotropic cross section. We find that in the case of small $\eta / s$, which corresponds to the case of a strongly coupled system, the energy density decays asymptotically as $\varepsilon_{\text {kin }} \propto t^{-4 / 3}$ which is what is expected in the ideal hydrodynamic limit in the case of a one-dimensional expansion, in agreement with [45]. For the cases of larger $\eta / s$ we find that a power law decay with a superimposed oscillation pattern is present. The thin dashed line in the figure corresponds to $t^{-4 / 3}$. These results are in good agreement with those of [54].

In the lower panel of Fig. 4 we plot the plasma temperature as a function of time; it is obtained by data shown in the upper panel of Fig. 4 by assuming a perfect gas equation of state which gives $T \propto \varepsilon_{\text {kin }}^{1 / 4}$ with proportionality constant being inversely proportional to the number of active degrees of freedom in the plasma. Our temperature is somehow larger than the one quoted in 54] because in the latter study both quarks and gluons
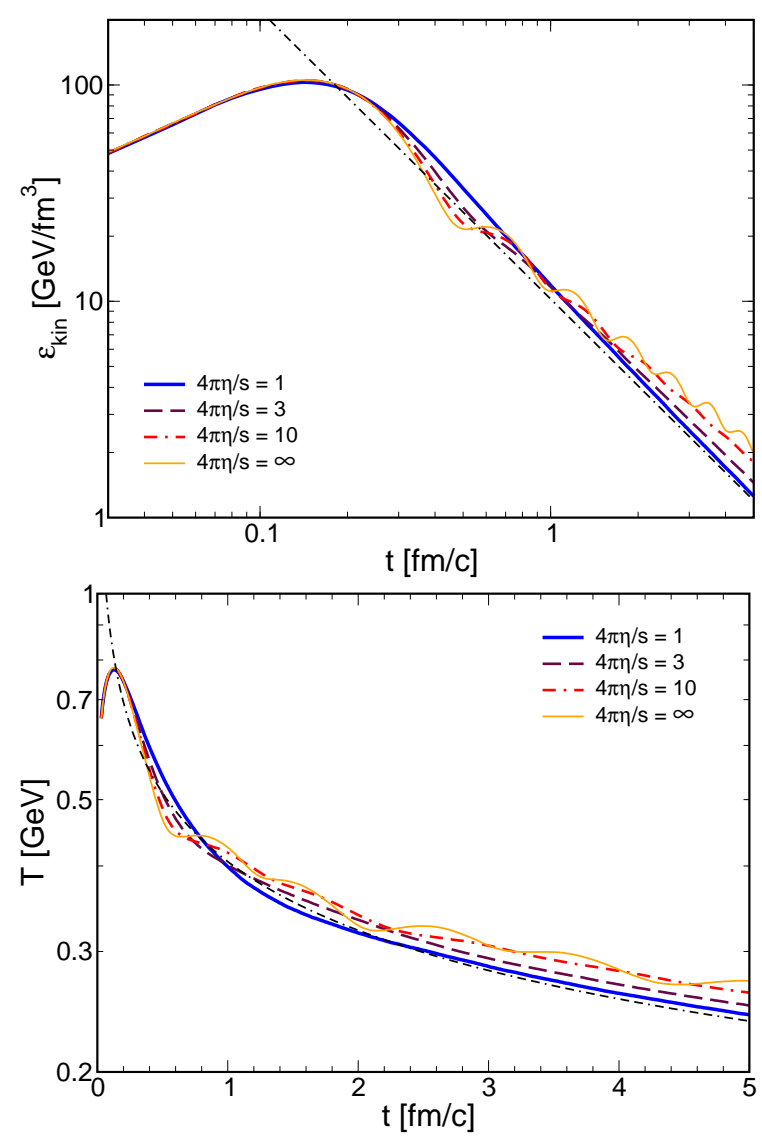

FIG. 4: Proper kinetic energy density (upper panel) and local temperature (lower panel). All the quantities are averaged in the central rapidity region $|\eta|<0.5$ and viscosity has been fixed using an isotropic cross section.

have been considered in the plasma, while in our case we only include gluons. The thin dashed line corresponds to $t^{-1 / 3}$ which is the power law decay expected in the case of a one dimensional expansion of a non viscous fluid.

In Fig. 5 we plot the gluon spectra at midrapidity $|y|<0.5$, for three different values of $\eta / s$. Upper panel corresponds to $4 \pi \eta / s=1$, middle panel to $4 \pi \eta / s=3$ and lower panel to $4 \pi \eta / s=10$. For each value of $\eta / s$ the spectrum at three different times is shown. The thin solid black line corresponds to a thermal spectrum, namely

$$
\frac{d N}{p_{T} d p_{T} d y} \propto p_{T} e^{-\beta p_{T}}
$$

the above relation describes a thermalized system in three spatial dimensions at the temperature $T=1 / \beta$. In the figure the thermal spectrum is computed by taking the temperature at $t=1 \mathrm{fm} / \mathrm{c}$ from data plot in Fig. 4. We find that for $4 \pi \eta / s=1$ the system efficiently thermalizes: in fact the spectrum at $t=1 \mathrm{fm} / \mathrm{c}$ is of the form (26) with temperature given (within a $2 \%$ ) by the result in Fig. 4 , as it is evident by comparing the thermal spectrum (black line) with simulation data (dot-dashed thin green line). For $4 \pi \eta / s=3$ and $4 \pi \eta / s=10$ the spectrum we obtain 

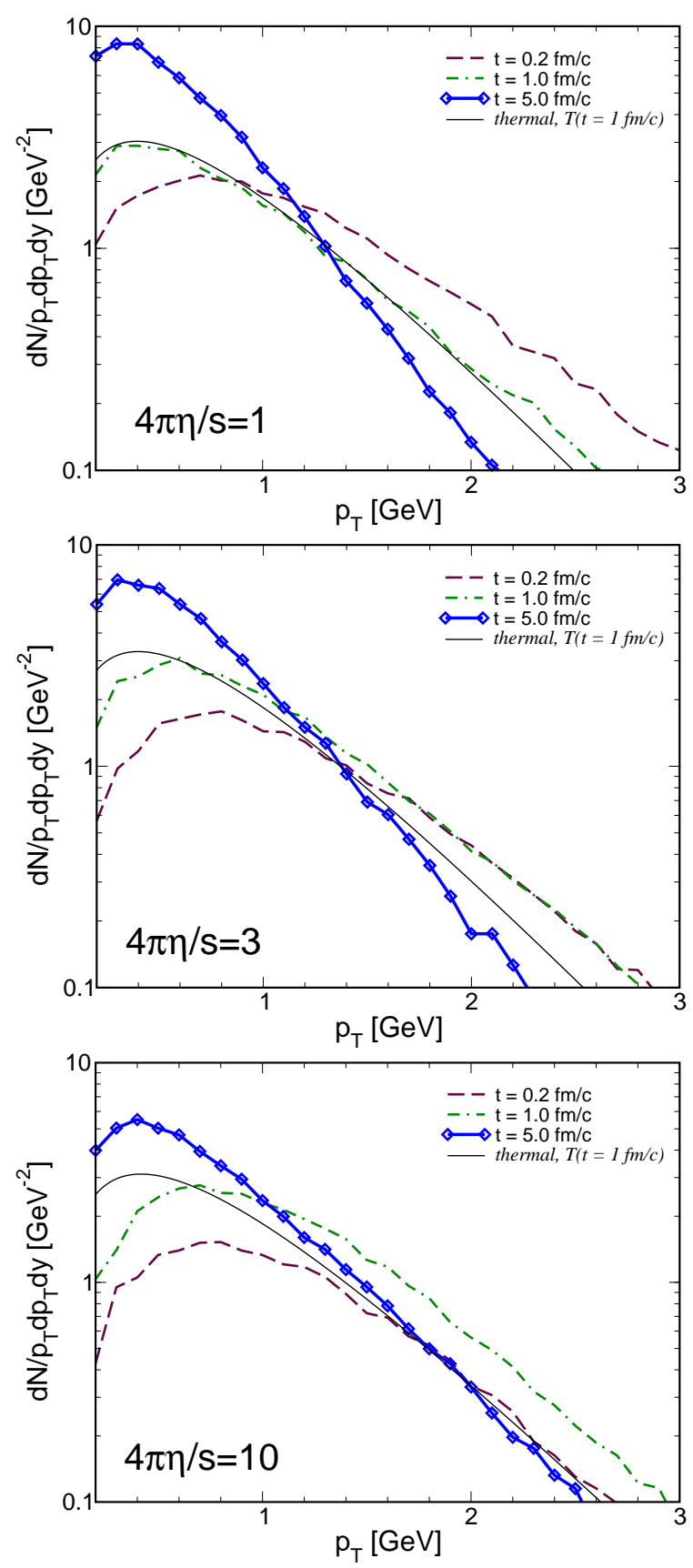

FIG. 5: Gluon spectra at midrapidity $|y|<0.5$, for three different values of $\eta / s$. Upper panel corresponds to $4 \pi \eta / s=$ 1 , middle panel to $4 \pi \eta / s=3$ and lower panel to $4 \pi \eta / s=10$. For each value of $\eta / s$ the spectrum at three different times is shown. Black thin solid line corresponds to the thermal spectrum in Eq. (26) at $t=1 \mathrm{fm} / \mathrm{c}$ with temperature from data in Fig. 4 .

in the numerical calculation is in some disagreement with the thermal spectrum at the temperature plot in Fig. 4 at $t=1 \mathrm{fm} / \mathrm{c}$, meaning the system is not completely thermalized in three dimensions. Moreover the very mild change in the slope of the spectrum we measure from

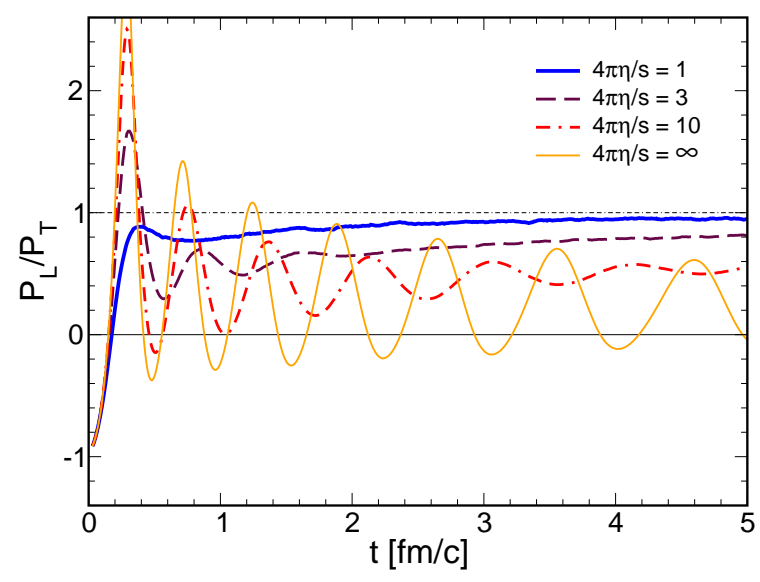

FIG. 6: Proper kinetic energy density (upper panel), local temperature (middle panel) and the ratio $P_{L} / P_{T}$ (lower panel) as a function of time. All the quantities are averaged in the central rapidity region $|\eta|<0.5$ and viscosity has been fixed using an isotropic cross section.

$t=1 \mathrm{fm} / \mathrm{c}$ to $t=5 \mathrm{fm} / \mathrm{c}$ shows that the system does not cool down efficiently in this case, as it is expected because the large viscosity implies that a large part of energy dissipates into heat and the system cools down more slowly than the case of small viscosity.

\section{B. Pressure isotropization}

In this subsection we discuss pressure isotropization in the case with a longitudinal expanding geometry. In Fig. 6] we plot the ratio $P_{L} / P_{T}$ where once again $P_{L}$ and $P_{T}$ correspond to the longitudinal and transverse pressure respectively. These quantities are computed cell by cell in the local rest frame of the fluid, then averaged in the rapidity range $|\eta|<0.5$. The initial longitudinal pressure at initial time is negative and $P_{L} / P_{T}=-1$ because at initial time the system is made of pure longitudinal chromoelectric field. On the other hand as soon as particles are produced, they give a positive contribution to the longitudinal pressure and the field magnitude decreases, eventually leading to a positive pressure. For all the value of $\eta / s$ we consider in our simulations we find that the time needed to the total longitudinal pressure to be positive is about $0.2 \mathrm{fm} / \mathrm{c}$. Moreover in the case $4 \pi \eta / s=1$ the strong interactions among the particles remove the initial pressure anisotropy quite efficiently and quickly: in this case $P_{L} / P_{T}=0.7$ within $0.6 \mathrm{fm} / \mathrm{c}$, then the ratio tends to increase towards 1 within the time evolution of the system. This would justify the use of viscous hydrodynamics with $\tau_{0} \approx 0.6 \mathrm{fm} / \mathrm{c}$ as commonly done.

On the other hand the larger the $\eta / s$ of the fluid the larger the oscillations of $P_{L} / P_{T}$, compare for example the cases $4 \pi \eta / s=1$ and $4 \pi \eta / s=10$ in Fig. 6. in the latter case $P_{L} / P_{T}$ experiences several oscillations which follow the alternation of maxima of $|E|$ (corresponding 

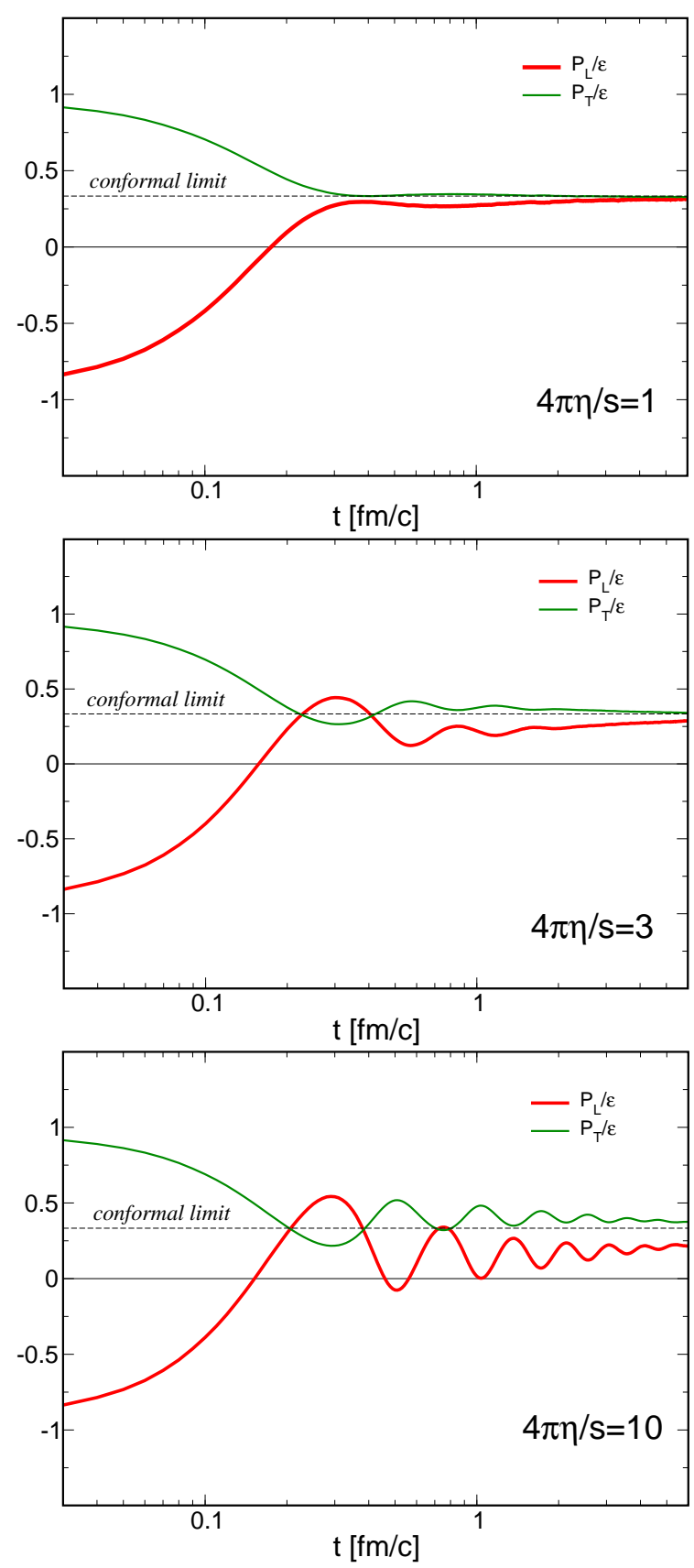

FIG. 7: Ratios $P_{L} / \varepsilon, P_{T} / \varepsilon$ against time for $4 \pi \eta / s=1$ (upper panel), $4 \pi \eta / s=3$ (middle panel) and $4 \pi \eta / s=10$ (lower panel). All the quantities are averaged in the central rapidity region $|\eta|<0.5$ and viscosity has been fixed using an isotropic cross section.

to minima of $P_{L}$ since the field gives a negative contribution to $P_{L}$ ) and zeros of $E$ (corresponding to maxima of $\left.P_{L}\right)$; also, at large times $P_{L} / P_{T}$ is quite smaller than 1 . For intermediate values of $\eta / s$ we still find some oscillation which become less important for smaller viscosities. On the same footing the asymptotic value of $P_{L} / P_{T}$ approaches 1 when the viscosity becomes small.
In Fig. 7 we plot the ratios $P_{L} / \varepsilon$ and $P_{T} / \varepsilon$ as a function of time. Here $\varepsilon$ corresponds to the total energy density, which takes into account energy density of both particles and field. We have shown results obtained for $4 \pi \eta / s=1$ (upper panel), $4 \pi \eta / s=3$ (middle panel) and $4 \pi \eta / s=10$ (lower panel). In Fig. 7 the red solid line corresponds to $P_{L} / \varepsilon$ and the thin green line to $P_{T} / \varepsilon$. The thin black dashed line corresponds to the conformal isotropic limit $\varepsilon=3 P$. One can compare the results of Fig. 7 with those of [9], where a classical Yang-Mills simulation with a $3+1 \mathrm{D}$ expanding geometry is considered. The spirit of our comparison with [9] is that, given we try to solve the same physical problem and we compute the same physical quantities, a comparison of the final results obtained within the two approaches is interesting even if the theoretical frameworks are different. In the weakest coupling case considered in [9], namely $g=0.1, P_{L}$ asymptotically relaxes towards zero, which we might obtain if we introduce a larger viscosity than the one we consider in the present study. On the other hand the case $g=0.5[9]$ produces $P_{L} / \varepsilon$ and $P_{T} / \varepsilon$ which lie in between our results for $4 \pi \eta / s=3$ and $4 \pi \eta / s=10$.

\section{Anisotropic cross sections}

In this subsection we study the effect of changing the microscopic cross section from isotropic to anisotropic. In our collision integral we achieve this by tuning the Debye screening mass in the two body cross section, leaving the value of $\eta / s$ fixed. In Fig. 8 we plot the time evolution of $P_{L} / P_{T}$ for four different values of $m_{D}$ for the case of $4 \pi \eta / s=1$ (upper panel), $4 \pi \eta / s=3$ (middle panel) and $4 \pi \eta / s=10$ (lower panel), and four different values of the Debye screening mass $m_{D}$. We remind that in our calculation the Debye mass is used as an infrared regulator of the differential cross section, and as a parameter which controls the anisotropy of the cross section: for very large values of $m_{D}$ the differential cross section is isotropic, while for small values of $m_{D}$ we get a forward peaked cross section.

We find that lowering $m_{D}$ for a given value of $\eta / s$ the plasma oscillations tend to be damped. This can be understood because according to [66] lowering $m_{D}$ while keeping fixed $\eta / s$ amounts to increase isotropization of the distribution function; as a consequence the conductive currents, which would sustain plasma oscillations in the late times evolution of the plasma, are damped. Nevertheless the effect on late time evolution of $P_{L} / P_{T}$ is quite mild.

\section{Chapman-Enskog versus Relaxation Time Approximation}

In this subsection we study the effect of shifting from the $\mathrm{CE}$ to RTA when we relate $\eta / s$ to the total cross section. It has to be noticed that our RTA does not cor- 

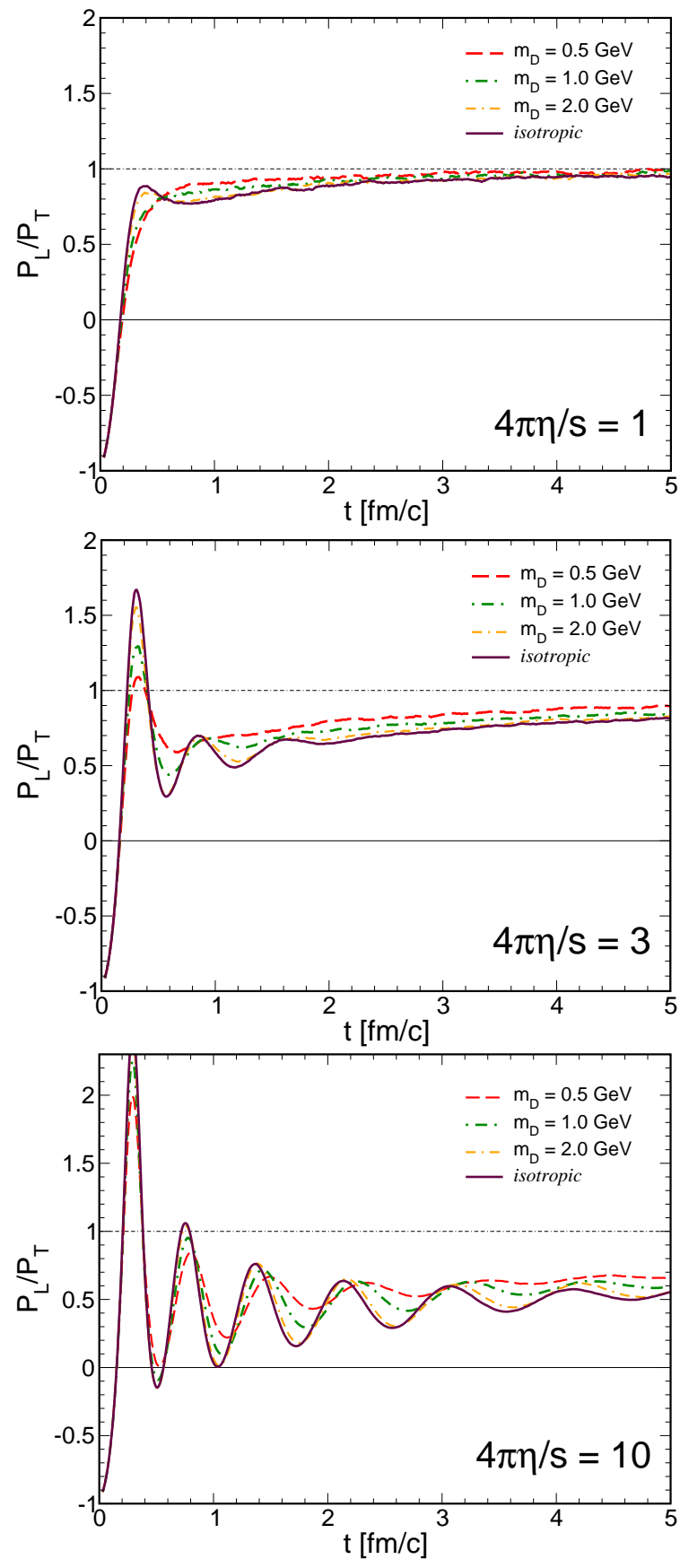

FIG. 8: Ratio $P_{L} / P_{T}$ versus time for several values of $m_{D}$ and $\eta / s$. All the quantities are averaged in the central rapidity region $|\eta|<0.5$. Upper panel corresponds to $4 \pi \eta / s=1$, middle panel corresponds to $4 \pi \eta / s=3$ and lower panel to $4 \pi \eta / s=10$.

respond to the RTA used in [54]: in fact in 54] RTA corresponds to an ansatz for the collision integral in the Boltzmann equation; on the other hand in our calculations, where we always solve the full collision integral in the Boltzmann equation, RTA refers only to an analytical equation which connects shear viscosity to microscopic
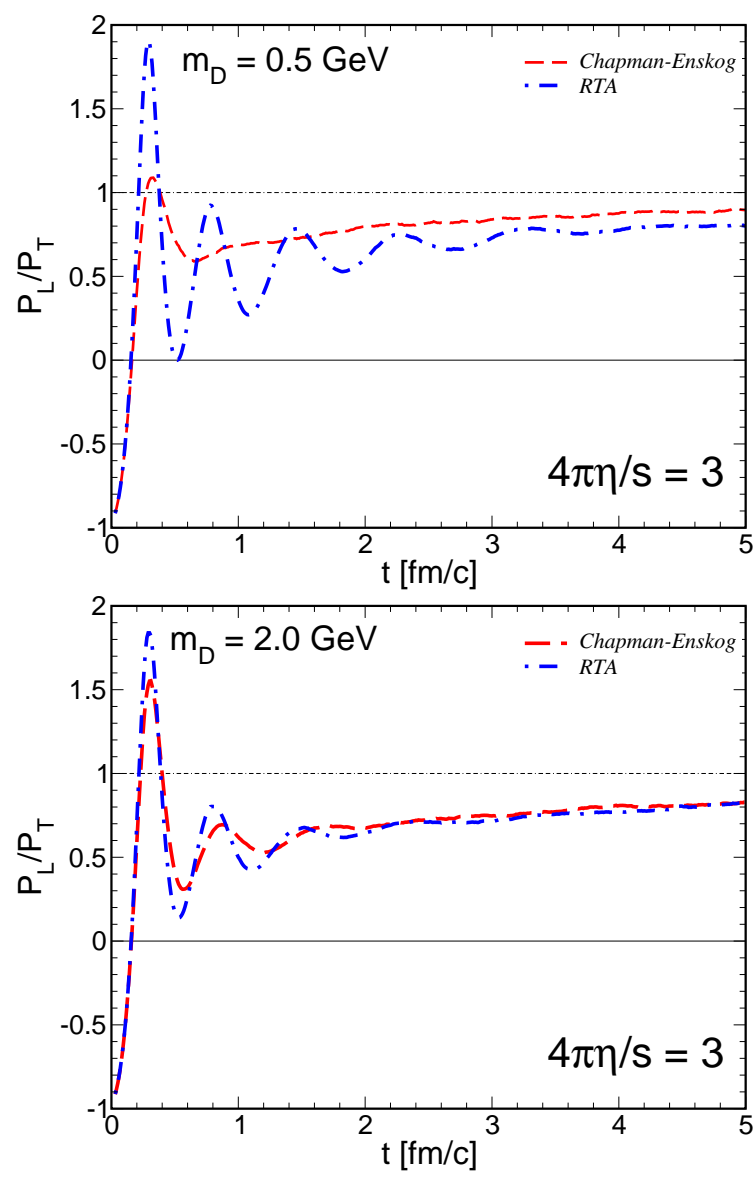

FIG. 9: Ratio $P_{L} / P_{T}$ versus time for $4 \pi \eta / s=3$ and for $m_{D}=$ $0.5 \mathrm{GeV}$ (upper panel) and $m_{D}=2.0 \mathrm{GeV}$ (lower panel). In both panels we compare the Chapman-Enskog result (dashed red line) with the Relaxation time result (dot-dashed blue line).

cross section 66]. We limit ourselves to a particular value of $\eta / s$, namely $4 \pi \eta / s=3$ which is the intermediate case we have considered in the previous subsections.

In Fig. 9 we plot $P_{L} / P_{T}$ as a function of time for two values of $m_{D}$ : in the upper panel we consider $m_{D}=$ $0.5 \mathrm{GeV}$ and in the lower panel $m_{D}=2 \mathrm{GeV}$. In each panel, the red dashed line corresponds to the CE formula while the dot dashed blue line to the RTA formula. In the case of $m_{D}=0.5 \mathrm{GeV}$ the oscillations of $P_{L} / P_{T}$ with $\mathrm{CE}$ are quite damped with respect to those of the RTA. This behaviour is easily understood by the results of [66] : as a matter of fact a given $\eta / s$ corresponds to a lower cross section for RTA, implying larger conductive currents which sustain plasma oscillations. In the case of $m_{D}=2.0 \mathrm{GeV}$ the behaviour of $P_{L} / P_{T}$ in RTA is similar to that in $\mathrm{CE}$; in fact in this case the cross section is mostly isotropic, and it is known that RTA cross section is quite close to the $\mathrm{CE}$ one. 


\section{SUMMARY AND CONCLUSIONS}

In this article we have reported about our results on the simulations of the Schwinger effect for color electric flux tubes, focusing on thermalization and isotropization of the fluid produced by the decay of the electric field in the tube. These simulations are important in the context of ultrarelativistic nuclear collisions, where flux tubes of strong color fields are expected to be produced in the early stage. According to the general understanding of high energy collision processes, this work is relevant both for heavy ion collisions, where a large number of tubes is expected, and for proton-nucleus and proton-proton collisions.

We have studied both the cases of flux tube decay in a static box and in a box with a longitudinal expansion. The flux tube decay is described by the Schwinger effect; the dynamical evolution of the field is assumed to be abelian so it satisfies Maxwell equations, and the dynamics of the fluid produced by the decay is studied by the relativistic transport theory coupled to the Maxwell equations. We have formulated transport theory in terms of a fixed value of $\eta / s$ rather than scattering amplitude; in this way we are able to describe the evolution of the fluid in terms of macroscopic quantities $(\eta / s)$ rather than insisting on a set of specific microscopic processes. The approach developed here, based on a stochastic solution of the relativistic Boltzman equation, has in perspective the advantage to be easily extended to $3+1 \mathrm{D}$ realistic simulations, allowing to study the possible impact of the early dynamics on observables like radial and elliptic flow [69]. Our study well matches [54] where the same problem has been studied. The main difference between the present study and [54] is that in the latter the relaxation time approximation is used to simplify the collision integral in the Boltzmann equation, while in our study we do not use such an approximation hence solving the relativistic kinetic equation with the full collision integral.

We have considered the case of a system with boost invariant longitudinal expansion, which is relevant for the case of simulation of early stages in heavy ion collisions. We have studied many aspects characterizing time evolution of the flux tube. In particular we have computed decay time of the color electric field, which we find to be a small fraction of $\mathrm{fm} / \mathrm{c}$; for large values of $\eta / s$ we have found plasma oscillations develop within $1 \mathrm{fm} / \mathrm{c}$ and persist for several fm/c in agreement with [54]; on the other hand for $\eta / s \leq 0.3$ plasma oscillations are very mild or even absent.

We have also computed the time evolution of the longitudinal to the transverse pressure ratio $P_{L} / P_{T}$. Given the particular initial field configuration and the absence of particle quanta at $\tau=0$ we have $P_{L} / P_{T}=-1$ at initial time; however longitudinal pressure becomes positive in $\tau \approx 0.2 \mathrm{fm} / \mathrm{c}$ because particles are produced by the Schwinger mechanism, giving a positive contribution to the pressure. For $\eta / s \leq 0.3$ we have found isotropiza- tion times $\tau_{\text {iso }} \leq 0.8 \mathrm{fm} / \mathrm{c}$; for larger values of $\eta / \mathrm{s}$ plasma oscillations cause the system to be less isotropic. Our results imply that for a fluid with $4 \pi \eta / s \leq 3$ isotropization time is less than $1 \mathrm{fm} / \mathrm{c}$. This would justify the use of viscous hydrodynamics with initial times $\tau_{0} \approx 0.6 \div 0.8$ $\mathrm{fm} / \mathrm{c}$ for $4 \pi \eta / \mathrm{s}=1 \div 3$. It remains to be understood if the oscillations of $P_{L} / P_{T}$ for $t \leq 1 \mathrm{fm} / \mathrm{c}$ affect some observable in ultrarelativistic heavy ion collisions. Our approach based on the stochastic solution of the Boltzmann equation is already set up to perform simulations starting from more realistic initial conditions and evolutions in $3+1$ dimensions, which will be useful to understand quantitatively the effect of such early times behaviour of physical quantities on observables.

We have also studied proper energy density and temperature evolution as well as particle spectra in the transverse plane for several values of $\eta / s$. We have found that in case of small $\eta / s$ the dynamics is very efficient in both removing the initial anisotropy and in getting a quick thermalization; in fact for $4 \pi \eta / s=1$ we find thermalization time $\tau_{\text {therm }} \leq 1 \mathrm{fm} / \mathrm{c}$ by comparing the particle spectrum at $t=1 \mathrm{fm} / \mathrm{c}$ with the thermal spectrum Eq. (26) where we put the temperature equal to the one we obtain in the simulation, see lower panel of Fig. 4. On the other hand, increasing $\eta / s$ results in a mismatch between the thermal and the actual spectra at $t=1 \mathrm{fm} / \mathrm{c}$, meaning thermalization time is larger in these cases.

To check the effect of microscopic details on the quantities we have computed, we have turned the microscopic cross section from isotropic to anisotropic by changing the value of the Debye screening mass in the two body cross section while keeping $\eta / s$ fixed. We have found that changing $m_{D}$ does not lead to significant changes in the evolution of physical quantities, besides small deviations in the early times evolution and some mild damping of field oscillations in the case of the largest $\eta / s$.

We have also considered the effect of turning from the CE to Relaxation Time Approximation (RTA) formula connecting the shear viscosity to the total cross section, still keeping the full collision integral in the Boltzmann equation. This is not exactly what has been done in 54 where RTA is used as an ansatz for the collision integral. We have found that in the case of a forward peaked cross section changing from CE to RTA affects the behaviour of $P_{L} / P_{T}$, increasing oscillations at early times. Nevertheless late times dynamics is almost unaffected by this change.

There are several points we have not considered in this study. We have neglected rapidity fluctuations in the electric field which would break boost invariance. Moreover in our description we have not included a longitudinal magnetic field at initial time which might modify sligthly the Schwinger amplitude [58]. Both fluctuations in rapidity and the initial chromomagnetic field are quite interesting ingredients to add to the present study and we plan to add them in future works. Moreover it would be of a certain interest to consider geometries with several flux tubes pinned on the transverse plane, hence pushing 
our calculations towards more realistic initial state conditions for heavy ion collisions as well as quantitative predictions of observables of phenomenological interest like multiplicities, particle spectra and collective flows. We will consider these developments in forthcoming publica- tions.

Acknowledgements. V. G., S. P., M. R. and F. S. acknowledge the ERC-STG funding under the QGPDyn grant. M. R. acknowledges discussions with T. Epelbaum, W. Florkowski and R. Ryblewski.
[1] T. Lappi and L. McLerran, Nucl. Phys. A 772, 200 (2006).

[2] F. Gelis and R. Venugopalan, Acta Phys. Polon. B 37, 3253 (2006).

[3] T. Lappi, J. Phys. G 35, 104052 (2008).

[4] F. Gelis, T. Lappi and L. McLerran, Nucl. Phys. A 828, 149 (2009).

[5] K. Fukushima and F. Gelis, Nucl. Phys. A 874, 108 (2012).

[6] H. Iida, T. Kunihiro, B. Mueller, A. Ohnishi, A. Schaefer and T. T. Takahashi, Phys. Rev. D 88, 094006 (2013).

[7] P. Romatschke and R. Venugopalan, Phys. Rev. D 74, 045011 (2006).

[8] H. Fujii, K. Itakura and A. Iwazaki, Nucl. Phys. A 828, 178 (2009).

[9] T. Epelbaum and F. Gelis, Phys. Rev. Lett. 111, 232301 (2013).

[10] J. Randrup and S. Mrowczynski, Phys. Rev. C 68, 034909 (2003).

[11] S. Mrowczynski, Acta Phys. Polon. B 37, 427 (2006).

[12] S. Mrowczynski, PoS CPOD 2006, 042 (2006).

[13] M. Attems, A. Rebhan and M. Strickland, Phys. Rev. D 87, no. 2, 025010 (2013).

[14] A. Rebhan, M. Strickland and M. Attems, Phys. Rev. D 78, 045023 (2008)

[15] B. Schenke, M. Strickland, C. Greiner and M. H. Thoma, Phys. Rev. D 73, 125004 (2006).

[16] P. B. Arnold, J. Lenaghan and G. D. Moore, JHEP 0308, 002 (2003).

[17] P. B. Arnold, J. Lenaghan, G. D. Moore and L. G. Yaffe, Phys. Rev. Lett. 94, 072302 (2005).

[18] P. B. Arnold, G. D. Moore and L. G. Yaffe, Phys. Rev. D 72, 054003 (2005).

[19] P. B. Arnold and G. D. Moore, Phys. Rev. D 73, 025006 (2006).

[20] A. Rebhan, P. Romatschke and M. Strickland, Phys. Rev. Lett. 94, 102303 (2005).

[21] A. Rebhan, P. Romatschke and M. Strickland, JHEP 0509, 041 (2005).

[22] L. Bellantuono, P. Colangelo, F. De Fazio and F. Giannuzzi, arXiv:1503.01977 [hep-ph].

[23] W. van der Schee, P. Romatschke and S. Pratt, Phys. Rev. Lett. 111, no. 22, 222302 (2013).

[24] Y. V. Kovchegov and A. Taliotis, Phys. Rev. C 76, 014905 (2007).

[25] M. P. Heller, R. A. Janik and P. Witaszczyk, Phys. Rev. Lett. 108, 201602 (2012).

[26] M. P. Heller, R. A. Janik, M. Spaliski and P. Witaszczyk, Phys. Rev. Lett. 113, no. 26, 261601 (2014).

[27] W. Heisenberg and H. Euler, Z. Phys. 98, 714 (1936).

[28] J. S. Schwinger, Phys. Rev. 82, 664 (1951).

[29] G. V. Dunne, In *Shifman, M. (ed.) et al.: From fields to strings, vol. $1 * 445-522$.

[30] F. Hebenstreit, J. Berges and D. Gelfand, Phys. Rev.
Lett. 111, 201601 (2013).

[31] F. Hebenstreit, J. Berges and D. Gelfand, Phys. Rev. D 87, no. 10, 105006 (2013).

[32] F. Gelis and N. Tanji, Phys. Rev. D 87, no. 12, 125035 (2013).

[33] G. C. Nayak, Eur. Phys. J. C 59, 715 (2009).

[34] G. C. Nayak, Electron. J. Theor. Phys. 8, 279 (2011).

[35] F. Cooper and G. C. Nayak, hep-th/0612292

[36] F. Cooper and G. C. Nayak, hep-th/0611125

[37] F. Cooper and G. C. Nayak, Phys. Rev. D 73, 065005 (2006).

[38] G. C. Nayak, Phys. Rev. D 72, 125010 (2005).

[39] A. Casher, H. Neuberger and S. Nussinov, Phys. Rev. D 20, 179 (1979).

[40] N. K. Glendenning and T. Matsui, Phys. Rev. D 28, 2890 (1983).

[41] A. Bialas and W. Czyz, Phys. Rev. D 30, 2371 (1984).

[42] A. Bialas and W. Czyz, Z. Phys. C 28, 255 (1985).

[43] A. Bialas and W. Czyz, Nucl. Phys. B 267, 242 (1986).

[44] M. Gyulassy and A. Iwazaki, Phys. Lett. B 165, 157 (1985).

[45] G. Gatoff, A. K. Kerman and T. Matsui, Phys. Rev. D 36, 114 (1987).

[46] H. T. Elze, M. Gyulassy and D. Vasak, Nucl. Phys. B 276, 706 (1986)

[47] H. T. Elze, M. Gyulassy and D. Vasak, Phys. Lett. B 177, 402 (1986)

[48] A. Bialas and W. Czyz, Acta Phys. Polon. B 17, 635 (1986).

[49] W. Florkowski, Acta Phys. Polon. B 35, 799 (2004).

[50] K. Bajan and W. Florkowski, Acta Phys. Polon. B 32, 3035 (2001)

[51] A. Bialas, W. Czyz, A. Dyrek, W. Florkowski and R. B. Peschanski, Phys. Lett. B 229, 398 (1989).

[52] A. Dyrek and W. Florkowski, Nuovo Cim. A 102, 1013 (1989).

[53] A. Bialas, W. Czyz, A. Dyrek and W. Florkowski, Nucl. Phys. B 296, 611 (1988).

[54] R. Ryblewski and W. Florkowski, Phys. Rev. D 88, 034028 (2013).

[55] W. Florkowski and R. Ryblewski, Nucl. Phys. A 931, 343347 (2014).

[56] W. Florkowski, Singapore, Singapore: World Scientific (2010) $416 \mathrm{p}$

[57] N. Tanji and K. Itakura, Phys. Lett. B 713, 117 (2012).

[58] N. Tanji, Annals Phys. 324, 1691 (2009).

[59] N. Tanji, Phys. Rev. D 83, 045011 (2011).

[60] Z. Xu and C. Greiner, Phys. Rev. C 79 (2009) 014904

[61] Z. Xu, C. Greiner and H. Stocker, Phys. Rev. Lett. 101 (2008) 082302

[62] E. L. Bratkovskaya, W. Cassing, V. P. Konchakovski and O. Linnyk, Nucl. Phys. A 856 (2011) 162

[63] G. Ferini, M. Colonna, M. Di Toro and V. Greco, Phys. Lett. B 670, 325 (2009) 
[64] S. Plumari and V. Greco, AIP Conf. Proc. 1422 (2012) 56

[65] S. Plumari, A. Puglisi, M. Colonna, F. Scardina and V. Greco, J. Phys. Conf. Ser. 420, 012029 (2013).

[66] S. Plumari, A. Puglisi, F. Scardina and V. Greco, Phys. Rev. C 86, 054902 (2012).

[67] M. Ruggieri, F. Scardina, S. Plumari and V. Greco, Phys Lett. B 727, 177 (2013).

[68] M. Ruggieri, F. Scardina, S. Plumari and V. Greco, Phys. Rev. C 89, no. 5, 054914 (2014).
[69] M. Ruggieri, A. Puglisi, L. Oliva, S. Plumari, F. Scardina and V. Greco, in preparation.

[70] A. Puglisi, S. Plumari and V. Greco, Phys. Rev. D 90, no. 11, 114009 (2014).

[71] L.D. Landau and E.M. Lifshitz, Electrodynamics of Continuous Media (Volume 8 of A Course of Theoretical Physics), Pergamon Press 1960.

[72] S. Plumari, G. L. Guardo, V. Greco and J. Y. Ollitrault, arXiv:1502.04066 [nucl-th]. 